\title{
PADRONIZAÇÃO DOS VALORES DO "TESTE DA LÁGRIMA DE SCHIRMER MODIFICADO" E DA "TONOMETRIA DE INDENTAÇÃO PELO MÉTODO DE SCHIÖTZ", EM CÃES DA REGIÃO DE JABOTICABAL - SP - BRASIL
}

\author{
STANDARDIZATION OF THE VALUES OF THE "SCHIRMER TEAR TEST" MODIFICATED AND OF THE' "S(WIOTY \\ TONOMETRY INDENTATION" FROM DOGSOF TIFE REGION OF JABOTICABAL. - SP - BRAZIL.
}

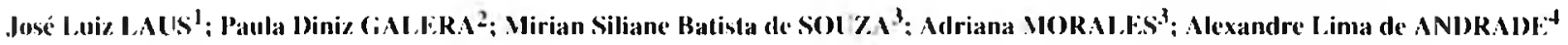

\begin{abstract}
RESLIMO

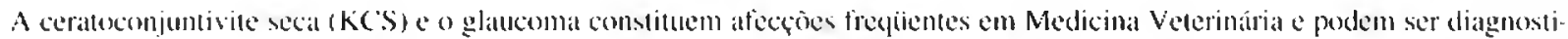

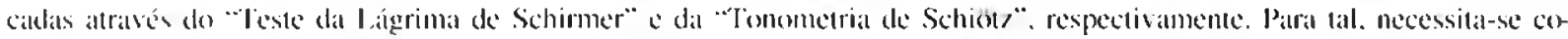

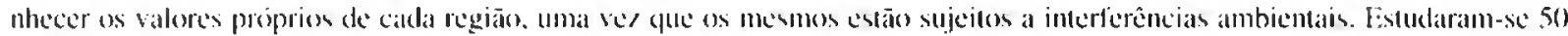

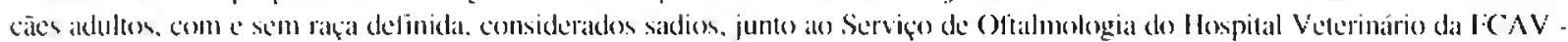

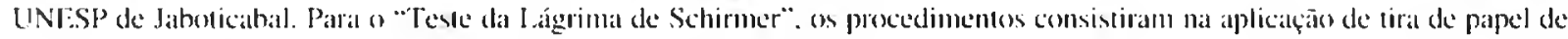

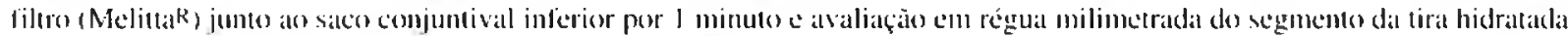

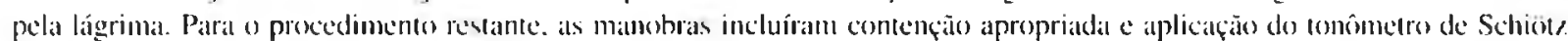
sobre o centro da córneal. previamente dessensibitizada com colírio anestésico. Os resultados mostranam valores médion de

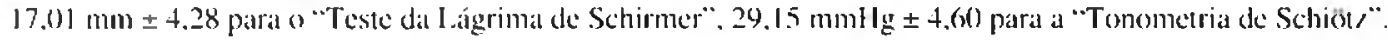

INTER WIOS: Teste de Schirmer: Tomometria de Schiorz. Cäs

\section{INTRODUÇÃO}

$O$ olho cuja função genérica é visual, lonecendo informaçōes do meio externo ao animal. é um órgão complexo. composto por estruturas altamente especializadas. A manutenção de sua homeostase, relacionada em parte aos anexos, encontra no aparcho lacrimal um de scus componentes mais significalivos.

O tïlme lacrimal contribui com proteção, oxigenaçāo e integridade da córnea. conservando-a umedecida. Dentre as afecçōes a cla relacionadas merece destaque a ceratoconjuntivite seca (KCS). paltologia de alta incidencia em cães, que resulta da hipoproduçấo lacrimal.

Quanto à cliopalogenial. há evidências de sua relação com afecções imunomediadas:. Algumas drogeas são também incriminadas 9.13. como tratamentos prolongados com sulfonamidas, atropina e fenazopiridina", além de anéstesicos rópicos?
Mediante procedimentos experimentais, constatou-se que a excisão das glândulas lacrimais principal e acessórials acarreta grandes danos à formação da lágrima, com desenvolvimento subseqüiente da KCS5

Contrariamente à KCS, a epífora é sintomatologia resultante de produção lacrimal excessiva. ou de distúrbios funcionais interagentes com o sistema de drenagem". Drenagem inadequada associada a moderado entrópio da porçāo medial da pálpebra inferior. lambém apontam-se como causas 5.10 .

Tanto epífora como KCS podem ser diagnosticadas pelo Teste da Lágrima, descrito inicialmente por Koster em 1900. Data de $19(0) 3$ a primeira modificaçấo do mélodo por Ono Schirmer, que preconizou at utilizaçäo de tiras de $0.5 \mathrm{~cm}$ de largura por $3.5 \mathrm{~cm}$ de comprimento e estabetecen que valores obtidos. inferiores a 1.5 mm de umidificaçäo. ém 5 minutos. para o homem. indicavam anormalidade do aparelho lacrimalt5. O procedimento consiske em inserir-se umal das

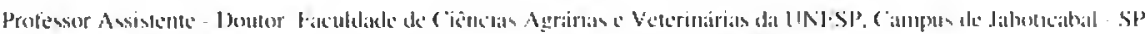

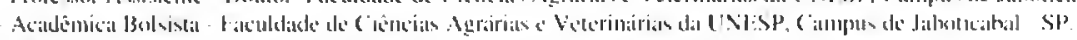

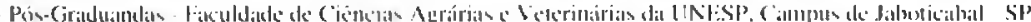

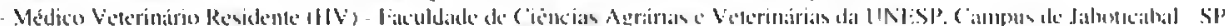




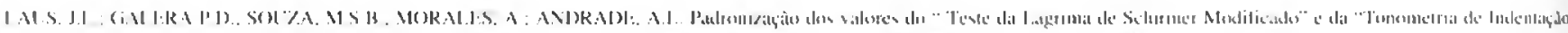

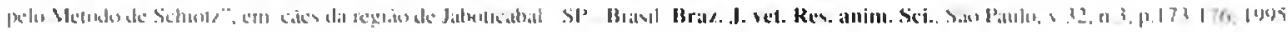

extremidades da tira de Schirmer. dobrada. junto alo saco conjuntival proximo a ângulo interno do olho. por 1 minuto e posterior avaliaçào. por memsuraçào da áreal umedecida. em régua milimetrada.

Alguns attores 1.7 .12 consideram a secreģäo lacrimal normal entre 15 c 20) $\mathrm{mm} / \mathrm{minuto.}$ ( )utros relacionam KCS a valores inferiores a $15 \mathrm{~mm} / \mathrm{minuto}$. Valores inferiores at 10 mm/minuto foram adotados parat confirmação dat conformidade 1.7.\%. Nio obstante. valores de $5-6$ mm/minuto podem extar presentes em animais normais 1 ?

Achados superiores a $15 \mathrm{~mm} /$ minuto, iguais e interiores a 10 mm/minuto, respectivamente. indicam secreçäo nomal. suspeição e KC S". Valores de ecro mm/minuto são indicaltivos de KCS gratce e. de 5 a $10 \mathrm{~mm} / \mathrm{minuto}$. são de calsos suspeiros 2.3. (onsideram-se fisiológicos valores entre 10-15 $\mathrm{mm} / \mathrm{minuto}$. Epíforal é referida para valores superiores a 25 mm/minuto2?

[tilizando-se papel de filtro Melittak. encontram-se valores médios normais situados en $20.73 \mathrm{~mm} / \mathrm{minuto} 1 \mathrm{t}$. Fin cães com sinais clínicos de KCS. na média, os valores assumem $3.15 \mathrm{~mm} / \mathrm{minuto}$. com desvio padrão de 7.0216 .

Testando diferentes lipos de papel nal aferição da gênese lacrimal, obscrvaram-se vantagens no emprego do papel MelittaR. (onsideram-se as facilidades de aquisição, o baixo custo ce os valores ques. yuando obtidos com o procedimento. habitualmente nào são divergentest. Os limites representam $1.40 \pm 1.5 \mathrm{~mm}: 0.72 \pm 1.0(0 \mathrm{~mm}: 18.00 \pm 3.40 \mathrm{~mm}: 29.89 \pm$ $6.72 \mathrm{~mm}$. respectia amente para a KCS. hipossecreção. secref̧ion normial è epillora.

A exemplo da KCS. o glancoma se traduy em problema corrente na ('línica Veterinárias. cujo diagnóstico, na maioria das vezes, só se dá quando da perda parcial ou total da visão. A lomometria é realizada de forma direta ou indiretax 11 . A primeira. simplista e subjetiva. é conduzida por pressão digilal do clínico sobre os olhos do paciente. Já a indireta ou instrumental, executadal por lécnicas de aplanação ou de indentação (Schiötz). é a mais empregada em nosso meio. Tal procedimento recpuer cooperação do paciente a ancstesial local. () animal é contido e o tonometro è descansaldo sobre 0 centro da córnear. sem que o clínico exerça pressão adicional. San realizadas 3 leituran consecutivas e a média entre clas.

Liscalas lidas entre 3 e 7 no tonônetro de Schiöt\% com 5.5 gramans de peso. indicam pression normal para o cão. Quando se empregam tahelas estimadas para valores humanos. a conversia é dada em 12.2 a $24.4 \mathrm{~nm} H \mathrm{Hg}^{0}$. A conversió da escala de leilura. para montge. difere entre on caĩes e o homem.

\section{MATERIAI. F MÉTOI)(}

\section{Animais}

Empregaram-se 50 cães adultos. machos c fêmeas. com ou sem raça definida. considerados sadios, oblidos no canil do Hospilal Velerinário da laculdade de Ciencias Agrárias e Veterinárias de Jaboticabal - L NE.SP e ciás alendidos junto ao Serviço de (Oftalmologia da entidade.

\section{PROTOCOI.OS EXPERIMENTAIS}

\section{"Teste da I ágrima de Schirmer"}

Avaliou-se o "Método de Schirmer" modificado. Para tanto. utilizou-se papel de filtro .Melitare preparado em tiras de 5 cm de comprimento por $0.5 \mathrm{~cm}$ de largura. () procedimentos compreenderam contenção do animal e avaliaçāo do método por aplicação da tira, dobrada a $0.5 \mathrm{~cm}$ em uma de suas extremidades. junto alo saco conjuntival. por I minuto. e subsequiente alerição da porção hidratada do papel. em régua milimetrada. () procedimento foi empregado para ambos os olhos.

\section{"Tonometria de indentação pelo método de Schiötz"}

Empregou-se anescesia locial, por instilação de colíro à basc de tetracaina. epinclirina é ácido borico*. contenção é retrollexàocervical.

Aplicou-se o lonomedro em posiçăo rerticall. com a balse cuidadosamente apoiada sobre o eentro da cormea. Valores ohtidos a partir dos proxedimentos foram alferides mediante keitura na escala de Sichoits. Paral cada animal. Lomaram-se três keituras a a média obtida entec elats. (omo no caso anterior. o cestudo foi conduzido nos dois olhos.

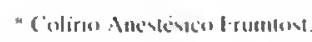

\section{RESUITADOS}

Pretendeu-se estudar o aparelho tacrimal ex a pressão intrat ocular.

O) resultados oblidos com o " "leste da lagrima de Schirmer" modificide compreendem valores médion de $16.83 \mathrm{~mm} / \mathrm{minuto} \pm 4.39$ para o olho direito e 17.18 mm/minuto \pm 4.19 para o contralateral. Para a Tonometria de Schiöt\% obtiveram-se $29.7 \mathrm{~mm} / \mathrm{Hg} \pm 4.8$ para o olho dircito e $28.6 \mathrm{~mm} / \mathrm{lg} \pm 4.4$ para 0 olho esquerdo. 


\section{DISCUSSÃO}

A ceratoconjuntivite seca (KCS) tem sua etiopatogenia. aventa-se, relacionada a doenças imunomediadas?. Paralelamente, tem sido relacionada a al gumas drogas $9.1,3$ e o seu emprego crônico. São exemplos as sullonamidas, a atropina e a fenazopiridina ${ }^{\%}$, além de anestésicos tópicos 5 . A extirpação das glândulas lacrimais principal c acessórias levam ao desenvolvimento da enfermidades.

Antagonicamente à KCS, a epífora é manifestação decorrente de fenômenos irritativos ou de distúrbios do sistema de drenagem da lágrimá. Moderado entrópio junto à porção medial da pálpebra inferior ${ }^{5} .10$ pode determinar o mesmo.

O Teste da Lágrima é realizado com fins diagnósticos tanto na epífora como na KCS. O mesmo sofreu modificações desde a sua propositura inicial, bem como os dados obtidos e suas interpretações.

A secreção lacrimal foi considerada normal para valores entre 15 e $20 \mathrm{~mm} / \mathrm{minuto} 1.12 .17$. Foram reportados valores inferiores a $10 \mathrm{~mm} / \mathrm{minuto}$ para os casos de KCS1.9. embora valores de $5-6 \mathrm{~mm} / \mathrm{minuto}$ possam estar presentes em animais normais 12 .

Achados de valores superiores a $15 \mathrm{~mm} /$ minuto, iguais a 10 $\mathrm{mm} /$ minuto e inferiores a $5 \mathrm{~mm} /$ minuto estão relacionados à secreção normal, suspeição e KCS, respectivamente.

Valores iguais ou próximos de zero $\mathrm{mm} /$ minuto relacionamse com KCS. Achados de 5 a $10 \mathrm{~mm} /$ minuto são de casos suspeitos. Consideram-se normais valores entre 10-15 $\mathrm{mm} / \mathrm{minuto}$ e indicadores de epifora os superiores a 25 $\mathrm{mm} / \mathrm{minuto}^{2,3}$.

Utilizando-se filtro de papel MelittaR. encontram-se valores médios normais situados em $20,73 \mathrm{~mm} / \mathrm{minuto}^{14} \mathrm{e}$. ainda. $17.7 \pm 3.93 \mathrm{~mm} / \mathrm{minuto} 16$. Ein cães com sinais clínicos da doença, a média apresentou-se com valores de 3,15 $\mathrm{mm} / \mathrm{minuto} \pm 7,02$.

Testando-se diferentes tipos de papel de filtro, inferiram-se, a partir dos valores obtidos com o papel Melittak, vantagens em relação aos demais 3.4.14.

Nos testes aqui realizados, tendo-se como animais de experimentação cães considerados clinicamente sadios, obtiveramse valores de $16,84 \mathrm{~mm} /$ minuto $\pm 4,39 \mathrm{em}$ olho direito e $17.18 \mathrm{~mm} / \mathrm{minuto} \pm 4,17 \mathrm{em}$ olho esquerdo, achados condizentes com os reportados na literatura3.4.14.16. Pode-se notar similaridade entre um olho relativamente ao seu contralateral. Muito embora o Teste ofereça boas condições de diagnóstico, não se imagina preterir o bom exame físico do paciente.

Relativamente ao glaucoma, ressalta-se a pouca frequência com que a tonometria indirela tem sido empregadal para diagnosticá-lo.

A leitura em Tonômetro de Schiötz, compreendida na escala entre 3 e 7 , com 5.5 gramas de peso, indica pressão normal no cão. Realizada a conversão, obtêm-se valores normais entre 12.2 a $24.4 \mathrm{mmHg}$.

Mediante os procedimentos realizados nesta pesquisa, puderam-se observar valores médios obtidos entre $29.7 \mathrm{mmHg}$ \pm 4.8 para o olho direito e $28.6 \mathrm{mmHg} \pm 4.4$ para o olho esquerdo. A exemplo do caso anterior. houve similaridade entre um olho e o seu contralateral.

Cabe ressaltar que a "Tonometria de Schiöty", embora admita possibilidades diagnosticas mais fidedignas, comparativamente à técnica digital. é munida. ainda. de certa imprecisão. Há fatores a considerar, como a mão do operador e. em alguns casos, a dificuldade na contenção adequada do paciente - prerrogativas importantes para a obtenção de valores fiéis, melhor conseguidos com a tonometria de aplanação.

Não obstante e consideradas tais assertivas. a técnica merece atenção, mormente nos casos suspeitos e carentes de diagnóstico diferencial. Ressalta-se comparativamente à técnica de aplanação que os limites de lidedignidade são menos alcançados quando se a emprega como procedimento semiotécnico. Por outro lado, as condições de custo implicadas dificultam a introdução da aplanação na rotina diagnóstica ofiálmica.

\section{CONCLUSÕES}

Frente às condições contidas nas proposituras iniciais, tornase licito admitir que:

- a utilização do "Teste de Lágrima de Schirmer Modificado" é eficaz para o diagnóstico de afecções do aparelho lacrimal:

- a utilizaçâo do papel de filtro Mellita ${ }^{\mathrm{R}}$ pressupōe técnica simples, exequíivel, fidedigna e de baixo custo:

- a "Tonometria de Indentação pelo Método de Schiötz" é procedimento de valor semiológico, apesar de suas limitaçōes.

\section{AGRAIECIMENTOS}

Os autores agradecem à Fundação de Amparo à Pesquisa do Estado de São Paulo (FAPESP - proc. 92/2018-6). pelo fomento dado ao estudo. 


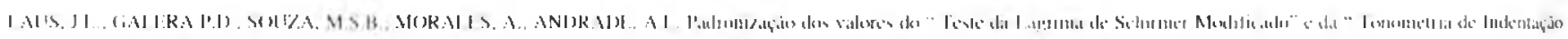

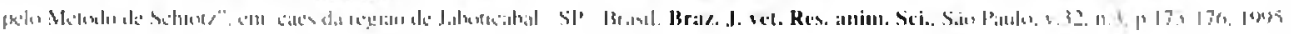

\begin{abstract}
SLMMINI

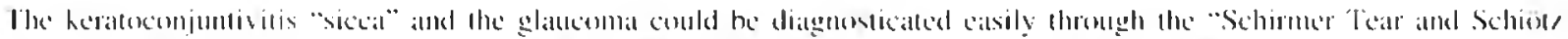

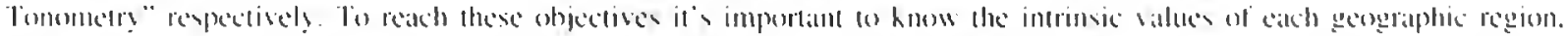

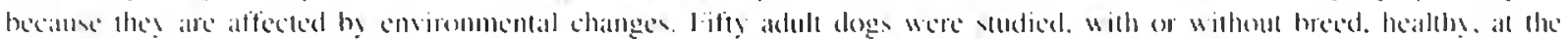

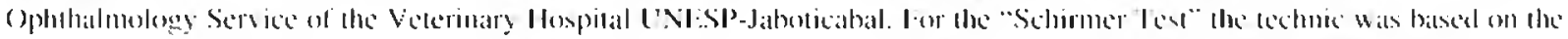

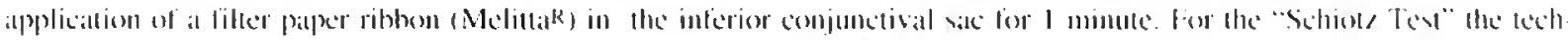

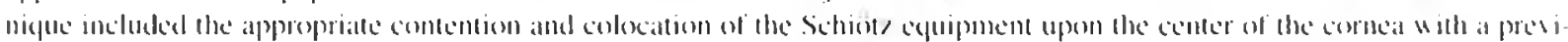

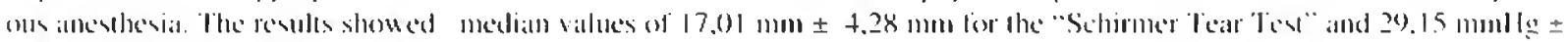
t.tol) for the "Sihiots. Tomomedry".
\end{abstract}

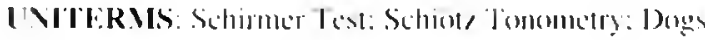

\title{
REFIRÊTCIAS BIBIIOCRÁFICAS
}

1-BARNETT. K.C.: SANSON JR. Diagnosis and treatment of keraloconjuntivilis sicca in dog. Veterinary Record. $5.120,11.14$, p..340-5. 1987.

2-BH:RNIS, W.( ). I:LRIDI:S, D.: BH:R.VIS IIII.HO. W.O. Manual de oftalmologia veterinária. Belo Horizonte. Universidade Federal de Minals (ierais. p.59-61. 1989.

3-CARVALIO). ('B.: KRALSI: A.; COSTA. P.R.S.: 1) (TRA, V. Teste da Lágrima de Schimer em cães. Cães e (iatos, 11.33, p.10-11,1991.

4-CARVALHO. C.B.: KRACSH: A.: (OSTA. P.R.S.: 1) "IRA, V. L tili/ação de papel de filtro como alternativa para avaliação do Tente de Schirmer em cães. Ciência Rural. 6.22, 11.3, p.281-3.1902

5-(il:1.ATT. K. N. Evaluation of katrs formation in the dog. using a modilication of the Schimer tear test. Journal of the American Veterinary Medical Association. 1.166. p. 368.197 .5

6-1HILPYR. I..C. Magrane's canine ophthalmology.

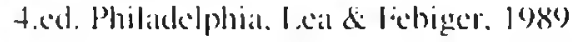

7-KASWAN, R.1.: MARTIN. ('.I.. I)AWE. I).I. Reumatoid factor determination in 50 doges with KCS. Journal of the American Veterinary Medical Issocialion. 1.18.3. n.10. p.107.3-5. 1983.

B-LOVIERKIN, L..(i.: BHILLIORN, R.W. clinicopathologic changes in primaly glatcoma in the cocker Spanicl. American Journal of Veterinary Rescarch. v.2\%, p.379, 1968.

9-IIORGA.N. R.V.: BACHRACH JR. A. KCS associaled with sulfomamide therapy in dogs. Journal of the American Veterinary Medical Association, 4.180, n.4. p.4.32-4. 1982.
10-PLEIFFI:R. R.L. Calibration of the Sehoue Tomometer for the normal caninc eyc. American Journal of Veterinary Rescarch. 1..38. p.1881-4.1977

II-PIIIFFER, R.L.: (iliLAT"I, K.N.. I978 apud HELPI:R L.C. Magrane's canine ophthalmology. 4.w. Philadelphiat. Lea \& Fobiger. p.77. 1989.

12-RUBIN, I.I.: AGUIRRE, (;. Clinical use pilocarpinc for KCS in dogs and cals. Journal of the American Veterinary Medical Association. v.151. 1.31,3-20, 1967.

1.3-SL ATTER, I).H.: BI.O)(F JR. KC S in dogs awrucialed with sulfomamide administration. Australian Veterinary Journal. v.54. 11.9. p.444-6. 1978.

I4-TLIDURY. IA.: SOLYA. WSB:BIAYONO, I : (iASTI: 1.. Uso do papel de filero Melilla na aleriçán do

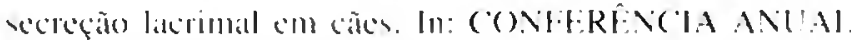

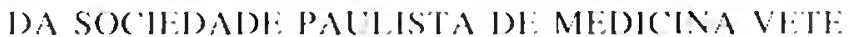
RINÁRIA, t6.. Sìn Palulo. |991. Anais. p.2

15-VAL(BHAN. 1): ASBURY. T. Oftalmologia geral Sino P’ulu, Alheneu. p.20-193. 1977

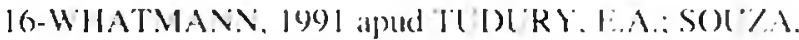

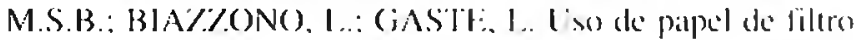
Melitta na allerição da secreçăo lacrimal em caides. In: ('O).

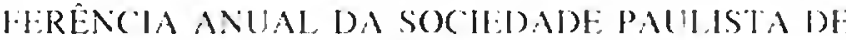

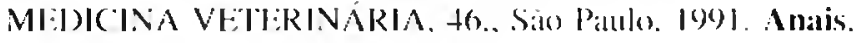
p. 2 .

17-WYMAN. M. Manual de oftalmologia de los pequeños animales. Barcelona. Salval. p.145-304. 1982.

Recebido para publicação em 14/03/94 Aprovado para publicação em 13/12/94 